\title{
Catastrophic antiphospholipid syndrome in the setting of mitral valve repair
}

\author{
Sami El-Dalati, MD, ${ }^{a}$ Matthew Suffoletto, MD, ${ }^{b}$ Ryan M. Rivosecchi, PharmD, ${ }^{c}$ and \\ David Kaczorowski, MD, ${ }^{\mathrm{d}}$ Pitsburgh, $\mathrm{Pa}$
}

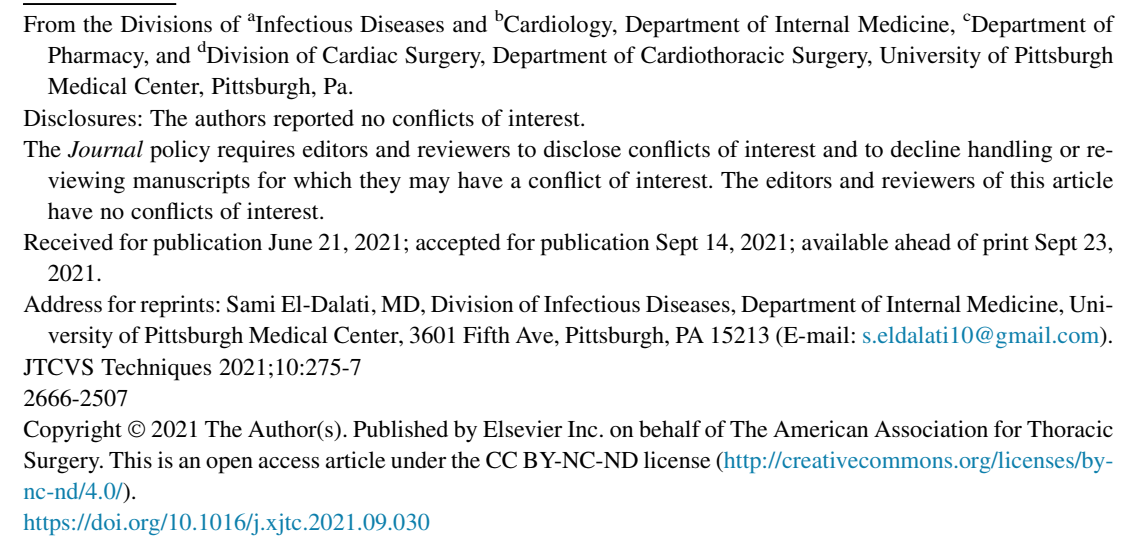

Video clip is available online.

\section{CASE REPORT}

A 58-year-old woman was admitted to the hospital after presenting with aphasia and left middle cerebral artery stroke. The patient had a history of stage III endometrial cancer status-post chemotherapy and brachytherapy as well as deep vein thrombosis and pulmonary embolism diagnosed $\sim 15$ months before presentation and managed with therapeutic enoxaparin. At that time, she underwent testing for antiphospholipid syndrome (APS) by lupus anticoagulant, which was negative.

On admission she reported a 2- to 3-week history of chills for which she took oral antibiotics. On hospital day 1 she underwent thrombectomy and recanalization of the middle cerebral artery. As part of her stroke evaluation, she underwent echocardiography that demonstrated a $1.1 \mathrm{~cm} \times 0.5 \mathrm{~cm}$ vegetation on the anterior leaflet of the mitral valve and a $0.8 \mathrm{~cm} \times 0.5 \mathrm{~cm}$ vegetation on the posterior leaflet with severe mitral regurgitation (Figure 1 and Video 1). Four sets of blood cultures yielded no growth. Additional admission laboratory studies were notable for a normal international normalized ratio and partial thromboplastin time.

Her case was discussed by the hospital's multidisciplinary endocarditis team. Considering her preceding

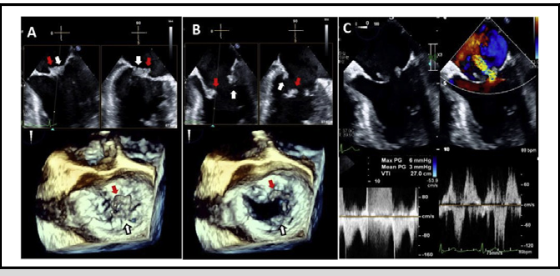

A and B, Mitral valve leaflet vegetations. C, Spectral and color Doppler TEE imaging.

CENTRAL MESSAGE

Antiphospholipid syndrome and catastrophic antiphospholipid syndrome are rare causes of endocarditis and complications of cardiac surgery. Consider testing in patients with culture-negative endocarditis or who develop thrombosis after cardiac surgery.

See Commentaries on pages 278 and 280 .

antibiotic therapy and symptoms she received antibiotic treatment for possible infectious endocarditis. In accordance with organization guidelines, the group recommendation was to proceed with mitral valve surgery to prevent further embolic complications and address the severe mitral regurgitation. ${ }^{1}$ Preoperatively, the patient was treated with intravenous unfractionated heparin and underwent contrasted computed tomography of the chest, abdomen, and pelvis, which was negative for pulmonary emboli, infection, or residual/metastatic endometrial cancer. She was evaluated by gynecologic oncology and deemed to be in clinical remission. Duplex ultrasonography of the bilateral lower extremities was negative for deep vein thrombosis. Given that the patient had undergone negative testing for APS within the past 15 months, was already on therapeutic anticoagulation with enoxaparin, had no other new thrombotic events, and presented with infectious symptoms while on oral antibiotics that could have caused falsely negative blood culture results, repeat testing for APS was not performed preoperatively. 

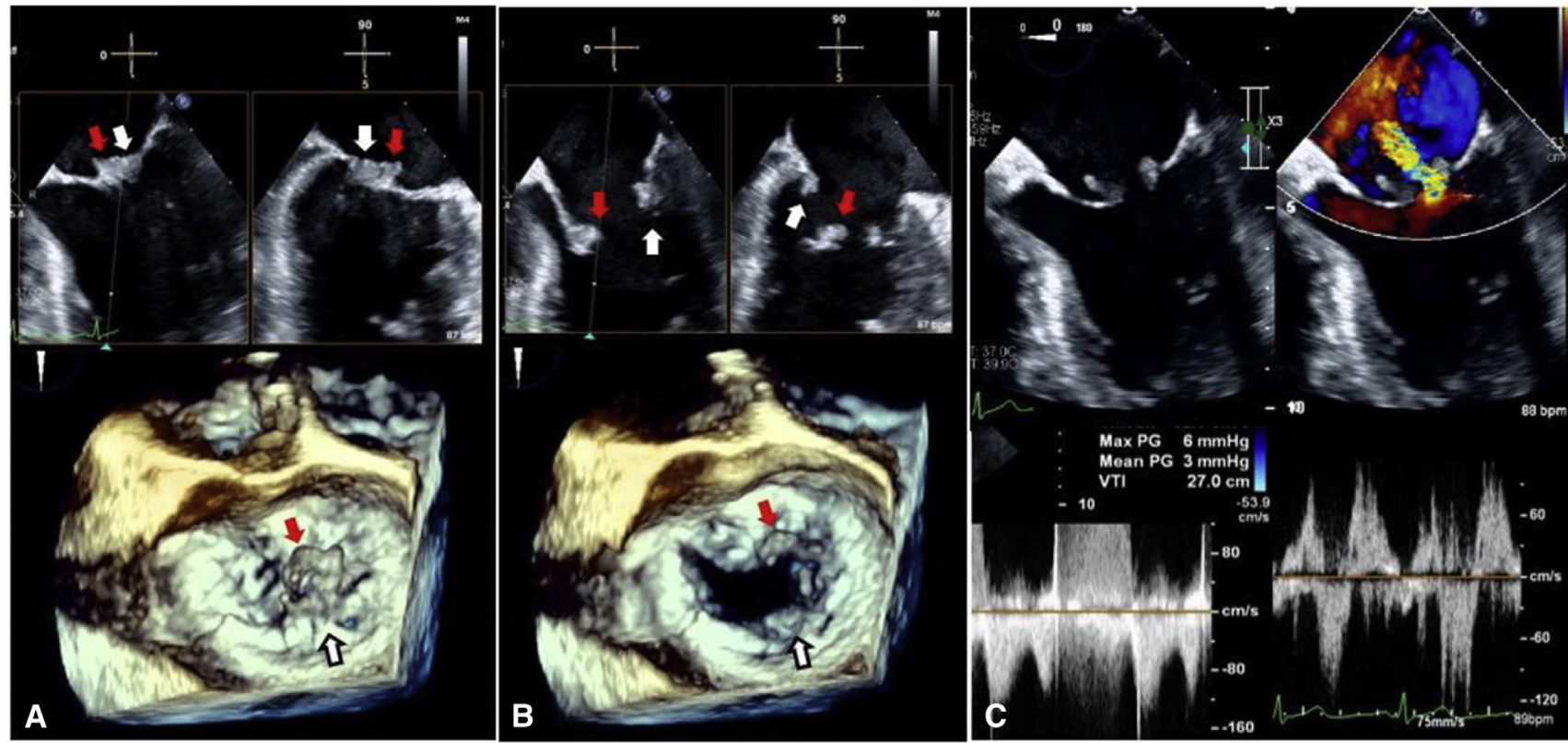

FIGURE 1. A, Mitral valve in systole, with red arrow indicating anterior mitral leaflet vegetation and white arrow indicating posterior leaflet vegetation. B, Mitral valve in diastole, with red arrow indicating anterior mitral leaflet vegetation and white arrow indicating posterior leaflet vegetation. $\mathrm{C}$, Color and spectral Doppler transesophageal echocardiography imaging. Upper panel demonstrates significant color Doppler mitral regurgitation jet at area of vegetation. Lower left panel demonstrates mildly elevated inflow mitral gradient secondary to vegetation and increased transvalvular flow due to degree of mitral regurgitation. Lower right panel demonstrates end-systolic flow reversal in the pulmonary vein due to significant mitral regurgitation.

On hospital day 22 she underwent uncomplicated mitral valve repair with a 28-mm Physio II (Edwards Lifesciences, Irvine, Calif) annuloplasty ring. Intraoperatively, the patient was noted to have mobile vegetations on both the anterior and posterior mitral valve leaflets that were debrided away, as well as destruction of the tissues between P2 and

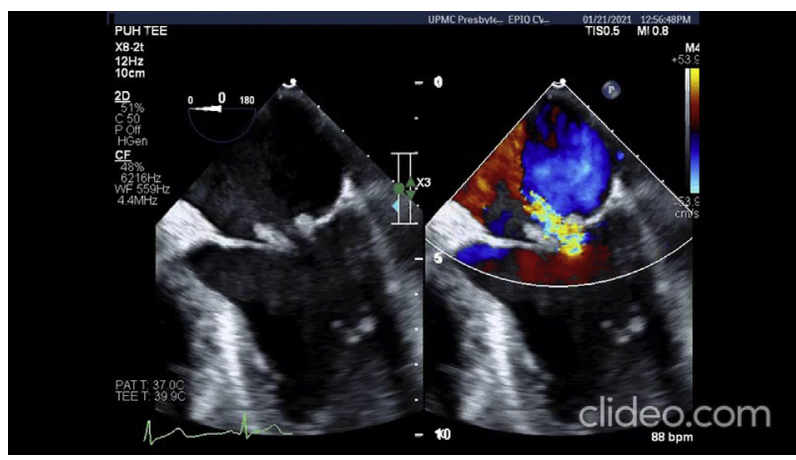

VIDEO 1. Simultaneous 2-dimensional and color Doppler imaging demonstrates bulky vegetation on both anterior and posterior mitral leaflets, involving primarily leaflet tips, associated with significant mitral regurgitation. Simultaneous biplane 2-dimensional imaging demonstrates the vegetation involving A2, P2, and P3 mitral valve scallops, further illustrated by 3-dimensional transesophageal echocardiography enface mitral valve imaging. Video available at: https://www.jtcvs.org/article/S26662507(21)00660-X/fulltext.
P3. The valve was repaired in accordance with literature demonstrating that patients with native mitral valve endocarditis who undergo repair have lower rates of postoperative bleeding, recurrent endocarditis, and reoperation when compared with patients who receive valve replacements. ${ }^{2}$ Cultures of the excised vegetations yielded no growth. On postoperative day (POD) 1 the patient's platelet count fell from a preoperative baseline of 150,000 to 64,000 and reached a nadir of 17,000 on POD2 at which time platelet transfusion was performed. Testing for heparininduced thrombocytopenia with platelet factor 4 antibodies and serotonin release assay was negative. During this period, the patient was anticoagulated with bivalirudin. On POD5 the patient was noted to have dusky bilateral extremities. Arterial duplex studies demonstrated thrombi in the left popliteal and bilateral radial and ulnar arteries; venous studies revealed acute deep vein thromboses in the right internal jugular and popliteal veins. Testing for antiphospholipid syndrome yielded a positive hexagonal lipid neutralization assay leading to a diagnosis of catastrophic APS (CAPS) on POD7. Between POD6 and POD22, the patient received anticoagulation therapy with argatroban before transitioning back to intravenous unfractionated heparin. Despite aggressive treatment including therapeutic anticoagulation, corticosteroids, intravenous immunoglobulin, and plasma exchange, the patient experienced new multifocal strokes confirmed by magnetic resonance 
imaging on POD16. On POD20, the patient was noted to have a 3 -g decrease in hemoglobin level with associated hypotension requiring transfer to the intensive care unit. No source of bleeding was identified on cross-sectional imaging. Between POD20 and POD31 the patient developed progressive uremia and altered mental status for which continuous renal replacement therapy was started on POD30. On POD31 she developed lactic acidosis and multiorgan failure. Computed tomography of the chest and abdomen revealed a new lobar pulmonary embolism and bowel ischemia with pneumatosis and portal venous gas. On POD32/hospital day 54, the patient died.

\section{COMMENT}

CAPS is a rare disease, more common in female patients, characterized by rapid onset of progressive microvascular thrombosis and multiorgan failure. CAPS represents the most severe form of APS, which itself is defined by the "occurrence of venous and arterial thromboses...accompanied by moderate thrombocytopenia, in the presence of antiphospholipid antibodies." 3 Thirty-eight percent to $50 \%$ of patients with APS and CAPS are found to have cardiac valvular lesions and CAPS is associated with $\sim 50 \%$ mortality. ${ }^{3,4}$ CAPS may be precipitated by surgeries, but this is just the fifth reported case of CAPS after cardiac surgery. ${ }^{4}$ In 2 reviews of valve surgeries performed in 66 patients with APS, there were no reported occurrences of CAPS. ${ }^{4,5}$
Nonbacterial thrombotic endocarditis secondary to APS was in the differential for the patient before cardiac surgery. However, this case was complicated by several factors, as discussed above in the case description and consequently, testing for APS was not ordered until POD4 and treatment was not initiated until POD7.

Given the associated significant morbidity, testing for APS should be considered in all patients with culturenegative endocarditis, particularly those undergoing evaluation for cardiac surgery because regression of valvular lesions with medical therapy has been reported. ${ }^{4}$ Thrombocytopenia and new thrombosis during the postoperative period should also prompt evaluation for CAPS.

\section{References}

1. Baddour LM, Wilson WR, Bayer AS, Fowler VG Jr, Tleyjeh IM, Rybak MJ, et al Infective endocarditis in adults: diagnosis, antimicrobial therapy, and management of complications: a scientific statement for healthcare professionals from the American Heart Association. Circulation. 2015;132:1435-86.

2. Harky A, Hof A, Garner M, Froghi S, Bashir M. Mitral valve repair or replacement in native valve endocarditis? Systematic review and meta-analysis. J Card Surg. 2018;33:364-71.

3. Colli A, Mestres CA, Espinosa G, Plasín MA, Pomar JL, Font J, et al. Heart valve surgery in patients with the antiphospholipid syndrome: analysis of a series of nine cases. Eur J Cardiothorac Surg. 2010;37:154-8.

4. Sukniam KB, Proia AD, Glower DD. Catastrophic antiphospholipid syndrome after cardiac surgery. J Card Surg. 2016;31:584-6.

5. Gorki H, Malinovski V, Stanbridge RD. The antiphospholipid syndrome and heart valve surgery. Eur J Cardiothorac Surg. 2008;33:168-81. 